\title{
Shear viscosity dependence on concentration of (polyethylene oxide - xanthan) pseudo plasticity polymer collide
}

Dependencia de la viscosidad de cizalladura en la concentración de pseudo plasticidad (oxido de polietileno - xantano) colisión de polímero

Autor:

Obaid K. A. (1)

Rassol S. R. (2)

Hussain A. J. (3)

Musa A. O. ${ }^{(4)}$

\section{ARTÍCULO DE INVESTIGACIÓN CIENTÍFICA Y TECNOLÓGICA}

How to cite this paper:

Obaid, K. A., Rassol, S. R, Hussain, A. J and Musa, A. O. Shear viscosity dependence on concentration of (polyethylene oxide xanthan) pseudo plasticity polymer collide, Babylon, Iraq. Innovaciencia Facultad de Ciencias Exactas, Naturales y Agropecuarias. 2018; 6(1) S1: 1-7.

\section{Reception date:}

Received: 15 August 2018

Accepted: 25 November 2018

Published Online: 28 December 2018

DOI:

http://dx.doi.org/10.15649/2346075X.482

Keywords:

PEO, Xanthan, Viscosity, Rheological

Properties.

\section{ABSTRACT}

Introduction: Its necessary to bear in mind that we life in the word increase industrialization, therefore we make many modifications to material to getting on best characterizations. The aim of this research is to Prepare new Pseudo Plasticity Polymer Collide. Materials and Methods: In the present paper effects of xanthan cellulose gum $(\mathrm{X})$ on rheological properties of polyethylene oxide polymer (PEO, 3000 Daltons) included different type of viscosity has been investigated by using the following parameters: (Spindle: no.1, Speed: $60 \mathrm{rpm}$ and Temperature: RT), different sort of viscosity is computed for a PEO that dissolved in distilled cold water with completely different various concentrations (0.1, 0.2 to 0.8$) \%$ $\mathrm{g} / \mathrm{mL}$ once and before adding ( 0.25 and 0.5$) \mathrm{g} \mathrm{X}$ for every concentration. Results and Discussion: The results show that all properties of density, shear viscosity, relative viscosity, specific viscosity, reduced viscosity, intrinsic viscosity, viscosity average mass and the effective molecular radius have been enhanced after the addition of xanthan. Conclusions: Addition of xanthan are often applied as thicker mixture in coating, oil drilling and pumping of fluids attributable related pseudo physical property.

\footnotetext{
Ministry of Education, Babylon of Educational Director,Babylon, Iraq.

2) Babylon University, College of Science, Chemistry Department,Iraq.

(3) College of Engineering Al-Musayab, University of Babylon,Iraq

(4) University of Babylon, College of Science, Department of Physics,Babylon,Iraq
} 


\section{INTRODUCTION}

PEO is a sort of Polyether. The major applications in textile, face powder (makeup) cosmetics, and agents of antifoaming, dyes and other chemical intermediates applications.

"Xanthan polyose, is one among the foremost vital microorganism polysaccharides created by genus xanthan bacteria genus campestris and by alternative xanthomonas species. This natural saccharide is an associate degree industrial biopolymer of valuable business due to rheology properties of xanthomonas collides, same high consistency at lower concentrations, pseudoplasticity and appear more stable over an honest variance of $\mathrm{T}, \mathrm{pH}$ values with concentration, this compound used also in food indus- tries, makeup, prescribed drugs, papers, paints, textiles and adhesive, additionally as inside oil and gas trade ${ }^{(1)}$. The aim of this study is fabrication of new blend material has pseudo plasticity can be used in oil drilling or pumping. The aim of this research is to Prepared new Pseudo Plasticity Polymer Collide.

\section{Theoretical Part}

Density (@) and also the shear viscosity of the PEO solutions before and once adding $\mathrm{X}$ for every concentration has been measured by the degree methodology and viscometer severally, moreover other kinds of viscosities have been computed by applying the below equations.

By the Jones-Dole equation, relative viscosity ( $\eta$ rel.) has been calculated ${ }^{(2)}$ :

$\eta$ rel. $=(\eta \mathrm{s} / \eta \mathrm{o})$

Where $\eta \mathrm{s}$ is shear viscosity, ๆo is the viscosity of pure water.

Specific viscosity ( $\eta \mathrm{sp}$.$) and reduced viscosity ( \eta$ red.) have been theoretically computed $\stackrel{(3)}{b}$ by:

$\eta$ specific $=(\eta$ relative -1$)$

$\eta$ reduced $=\eta$ specific $/$ concentration

Intrinsic viscosities are obtained by reading the intersection at the coordinate axis line as (c) goes to dilution of between reduced viscosity and the concentrations, that diagrammatically accepted value of intrinsic viscosity before and when $\mathrm{X}$ additions. Intercept values for those curves are shown in Table (1). Intrinsic viscosity may be in theoretically calculated by the equation of Philippoff as:

$\eta$ rel. $=[1+[\eta]]^{8}$

The relation between intrinsic viscosity $[\eta]$ and relative viscosity is calculated by Arrhenius equation ${ }^{(4)}$ as :

$\ln \eta$ rel. $=[\eta] \mathrm{C}$

Viscosity average mass has been determined by equation ${ }^{(5)}$

$[\eta]=\mathrm{k} \mathrm{M}^{\mathrm{a}} \mathrm{v}$ 
(k) and (a) are square measure constants rely upon the kind of compound, for xanthan. All values are obtained from standard Tables then, $(\mathrm{a}=0.89, \mathrm{k}=2.855 \times 10-4)[6]$.

The radius of effective molecules (r) was calculated_(7) by:

$\eta$ rel. $=1.12+6.33 \times 1024 \mathrm{r} 3 \mathrm{~cm}$

slope $=6.3 \times 1024 \mathrm{r} 3$

$\mathbf{r}_{\mathrm{o}}=\sqrt[3]{\text { slope } / 6.3^{* 10^{24}}}$

The slope term is obtained from eqn.(8) which gives the relationship between relative viscosities and concentration.

\section{Practical Part:}

PEO and $\mathrm{X}$ have been used in this article.

\section{Preparation of Solutions:}

PEO (3000 Daltons) with purity (98.98\%) and X with purity $(99.97 \%)$ of high viscosity. PEO collides are ready dissolving a known weight of $\mathrm{PEO}$ powder at $\mathrm{H} 2 \mathrm{O}$ in $(0.5 \mathrm{~L})$ stirring without for $(50 \mathrm{~min})$. The PEO concentrations $(0.1,0.2$ to 0.8$) \% \mathrm{~g} / \mathrm{L}$, therefore $\mathrm{X}$ further with percentage weights grams added to each PEO concentrations. The following solution stirred for fifteen minutes till the blend became homogenous. Also, the viscometer has the following specifications (Spindle: no.1, Speed: $60 \mathrm{rpm}$ and Temperature: RT $\left(20^{\circ} \mathrm{C}\right)$ by shear stress technique.

\section{RESULTS AND DISCUSSIONS}

\section{Physical science properties}

Density for each PEO solution before and after addition $\mathrm{X}$ were measured at RT. Density will increase when concentration was increased as in (Fig.1), results of were attributed to enlarged mass of solution. Furthermore, swelling created within the compound chains because of the solubility in $\mathrm{H}_{2} \mathrm{O}$ and better mass of particular polymers ${ }^{(8)}$.

Figure 1. Density verses concentrations of pure and collides.

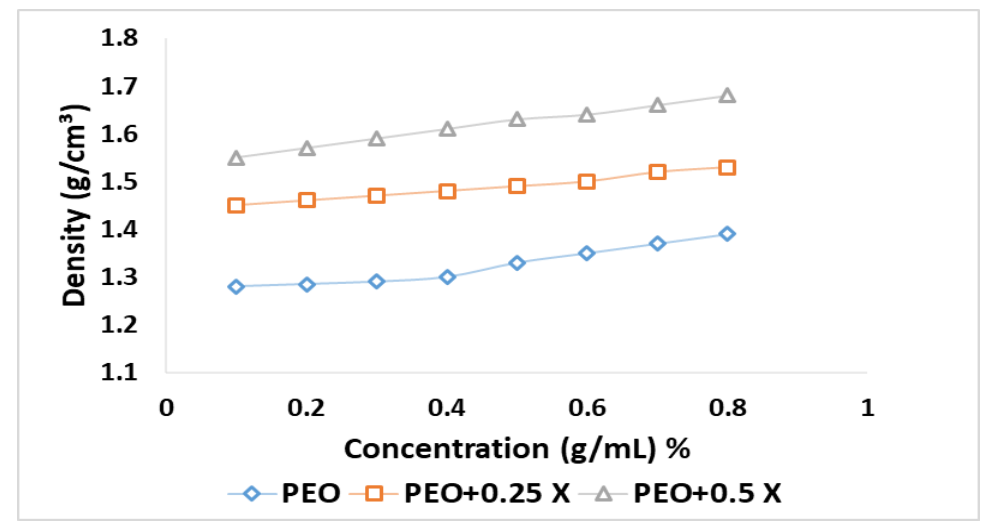


Shear viscosity increases as in (Fig.2). This behavior is related to Vander Waals bonding of water at gas sites, this lands up in sheaths of salvation and increase among the scale of the molecules.

The value of shear viscosity increased once $\mathrm{X}$ addition has started, the explanation is due to attractive forces between molecules of the solution once the addition, thus usually often leads in particle branching in combination so occupying a bigger volume. This can be often leading to make increasing in the values of shear bodies $\stackrel{(9)}{ }$ :

Figure 2. Shear viscosity due to concentrations of pure and collides.

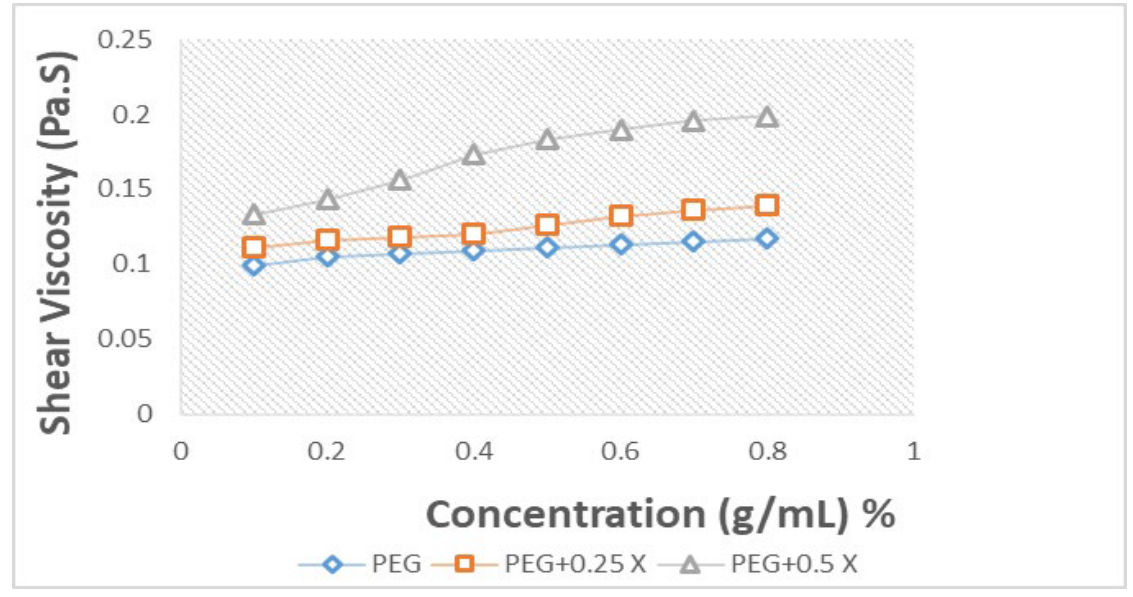

Each viscosity is sorted for the compound before and once $\mathrm{X}$ addition of square measure appearing by the pattern of equations (1 to 3). Depending on these PEO viscosity solutions and additives on the increasing of concentrations are clear in Figures (3 to 5). Viscosities unit severally possessing the same behaviors of shear viscosity as a result of they are being from it. Adding X created improvement for these viscosities as a result of the viscosity describes internal resistance of fluid among molecules therefore once adding X there'll be additional molecules, extra tights between molecules cause a further contribution to the shear stress $\stackrel{(10)}{\text {. }}$

Figure 3. The relative viscosities verses concentration of pure and collides.

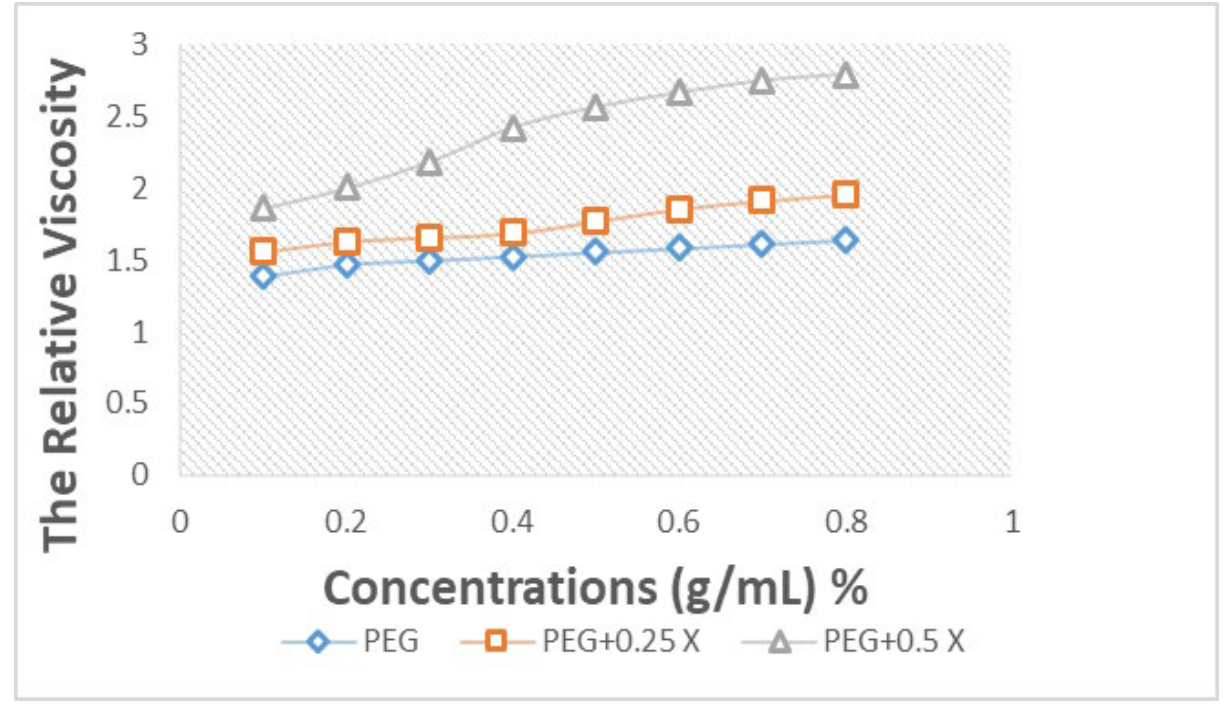


Figure 4. The specific viscosities verses concentration of pure and collides.

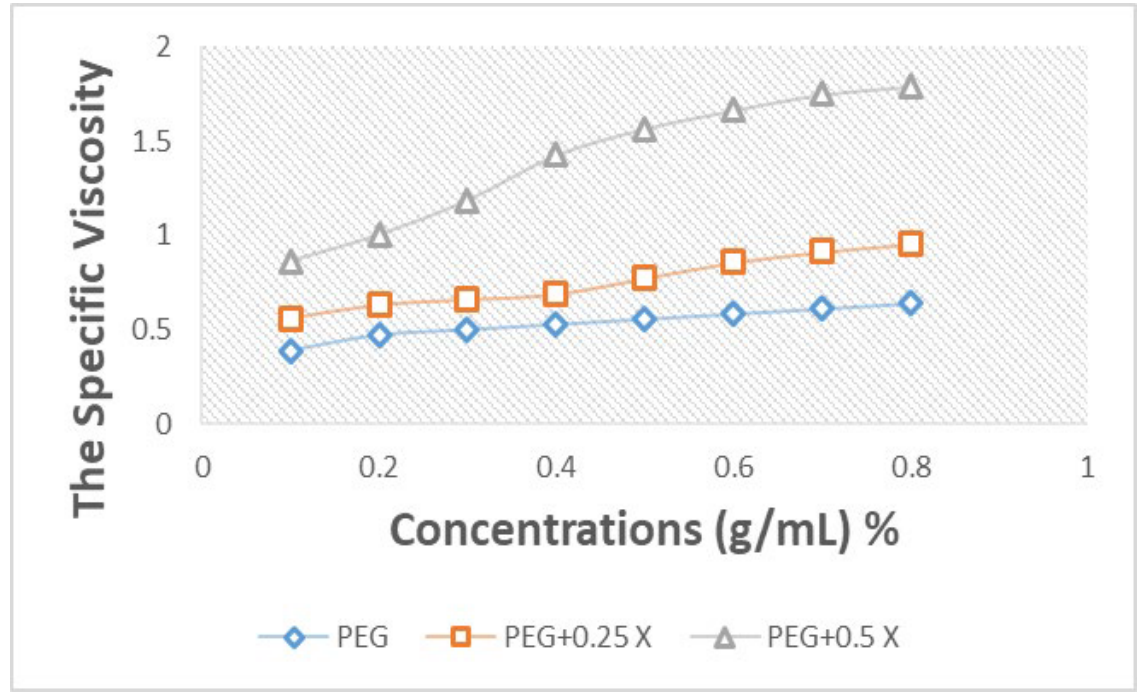

Figure 5. Reduce viscosity due to concentration of pure and collides.

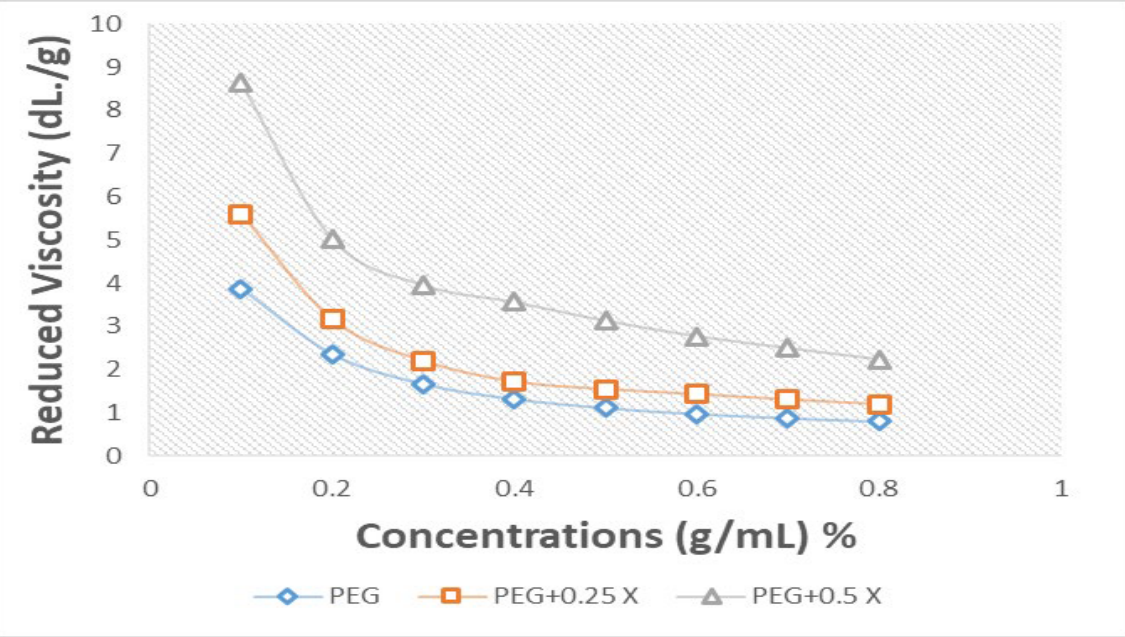

Intrinsic viscosities for each sample were calculated by drawing a graph between reduced viscosities versus concentrations. The slope extrapolation of concentrations approaches to zero up to intrinsic viscosity values, as appear in Fig. (5). Fig. (5) which shows the intersection point with (y-axis) for PEO with two cases of the addition of $X$. Intrinsic viscosity represent the molecule effective volume of a particle ${ }_{-}^{(11)}$ [11]. Theoretical intrinsic viscosities have been computed by Philippoff equation and Arreh. equation. The calibration between experimental values and theoretical were obtained values by pair of equations shown in Table (1).

Table (1) Intrinsic viscosity calibrations of polymer and its additives.

\begin{tabular}{cccc}
\multirow{2}{*}{ Polymer } & \multicolumn{3}{c}{ Intrinsic body[n](mL/g) } \\
\cline { 2 - 3 } & \multicolumn{2}{c}{ Theory } & Practicle \\
\cline { 2 - 4 } & Arrh. & Philip. & 6.1 \\
PEO & 6 & 6.2 & 7.4 \\
PEO+0.5g X & 7.3 & 7.5 & 8.6 \\
\hline
\end{tabular}


Results show a decent agreement between experimental and theoretical viscosity values. This description of viscosity has been attributed to structural amendment related to a liquid compound resolution and possibly indicating trap interaction $\stackrel{(12)}{ }$.

Viscosity average $\mathrm{Mw}$ before and once $\mathrm{X}$ adds is computed by Appling eqn. (6). Values of viscosity are taken through an experiment from the known values of intrinsic viscosity and therefore (k) and (a) are constants obtained upon the compound sort as within the Table no. (2). (k) and (a) obtained from standard Tables [13]. The values of (k) also calculated by applying eqn. (6). Comparison between calculated value of viscosity average $\mathrm{Mw}$ obtained by Philippoff eqn. and Arrhenius equations and experimental values by intrinsic viscosity as in Table (2).

Table (2) Calibration of (Mv) of pure polymer and its additives.

\begin{tabular}{lcccc}
\multirow{2}{*}{ Polymer Sorts } & $(\mathbf{K})$ constant $\times \mathbf{1 0}^{-\mathbf{4}}$ & \multicolumn{2}{c}{ Theory } & \multirow{2}{*}{ Practicle } \\
\cline { 3 - 4 } & 2.85 & Arrh. & Philip. & 5 \\
\hline PEO & 3.67 & 5.1 & 4.9 & 6 \\
PEO $+0.25 X$ & 3.89 & 6.3 & 5.9 & 7 \\
PEO $+0.5 \mathrm{~g} X$ & 7.17 & 6.89 & 7 \\
\hline
\end{tabular}

Results additionally show that viscosity average molecular weights area unit increasing when the addition of $\mathrm{X}$ is achieved, the rationale is that mass is outlined because the product of the mass of the chemical compound by the degree of chemical change $\stackrel{(13)}{ }$
The radius ( $\mathrm{r}$ ) for the top concentrations increased when $\mathrm{X}$ is added as in Table (3). Its known that the viscosity is the relation between shear stress and shear rate therefore the rationale for these increments is generated gel compounds because the pseudo plasticity phenomena, so modify the number of chains that appear as random coils, then increasing the effector molecules radius $\stackrel{(14)}{~}$.

\section{Table (3) The radius (r) for each concentration of pure polymer and its additives.}

\begin{tabular}{cc}
\multicolumn{2}{c}{$(\mathbf{r o})(\mathbf{c m})$} \\
\hline Polymer Sorts & Conc. $\times 10^{-25}$ \\
PEO & 1.9787 \\
PEO + 0.25g X & 7.65 \\
PEO+ 0.5g X & 8.69 \\
\hline
\end{tabular}

\section{CONCLUSIONS}

1. Adding $\mathrm{X}$ to $\mathrm{PEO}$ increased its density.

2. Increasing of concentrations extend the viscosity, therefore are often applied as thicker mixture in coating, oil drilling and pumping of fluids attributable related pseudo physical property. 


\section{REFERENCES}

1. Zorana Z R, Bojana $\check{Z}$ B, Jovana A G, Siniša N D, Jelena M D, "Effect of the Initial Glycerol Concentration in the Medium on the Xanthan Biosynthesis", Original Scientific Paper, 2014; 45: 239-246.

2. Biswal R, Singh P "Characterization of Carboxymethyl Cellulose and Polyacrylamide Graft Copolymer", Carbohyd Polym., 2004; 57:379-387.

3. Abdul-Kareem J, Safa A," Study the Effect of Adding PVA on Some Physical Properties of CMC Polymer as Aqueous Solutions", Advances in Physics Theories and Applications, 2012; 5:1-8.

4. Abdul-Kareem A, Rawaa M, Burak Y," Study the Rheological and Mechanical Properties of PVA/ NH4Cl by Ultrasonic", Chemistry and Materials Research, IISTE, 2011; 1,1:20-26.

5. Illiger R, Rao P, Demappa, T, “ Miscibility Studies of HPMC/PVA Blends in Water by Viscosity, Density, Refractive Index and Ultrasonic Velocity Method, Carbohydrate Polymer", 2008; 15, 3: 779-782.

6. Asia K," Effect of Adding Polyacrylamide on Some Physical Properties of Polyvinal Alcohol and its Ability for Industrial Applications", M.Sc. thesis, Babylon University, College of Science, Department of Physics, 2015.
7. Gurul S, Prasad P, Shivakumar R, Rai K," Studies on The Compatibility of Pullulan - Carboxymethyl Cellulose Blend Using Simple Techniques", Malaysian Polymer Journal, 2008; 3, 2: 13-23.

8. Abdali K," Enhancement of Some Physical Properties of Polyethylene Glycol by Adding Some Polymeric Cellulose Derivatives and its Applications", Ph.D. thesis, Babylon University, College of Science, Department of Physics, 2015.

9. Palani R, Kalavathy S, “ Volumetric Compressibility and Transport Studies on Molecular Interactions of Mono, Di and Tri Saccharine in Aqueous Sodium Butyrate Mixtures at $303.15 \mathrm{~K}$ ", Advances in Applied Science Research, 2011; 2, 2: 146-155.

10. Benchabane A, Bekkour K, "Rheological Properties of Carboxymethyl Cellulose Solution", Springer Journal, 2008; 286, 10: 1173-1180.

11.Jassim H, "Effect of Polyacrylamide on Physical Properties of Carboxymethyl Cellulose", M.Sc. thesis, Babylon University, College of Education for Pure Sciences, 2014.

12. Irina L, Maria B, Simona M, "Intrinsic Viscosity of Aqueous Polyvinyl Alcohol Solutions", Revue Roumaine de Chimie., 2009; 54: 981-986.

13. Ahmed S, "Study of The Physical Properties of Polymer Carrboxymethyl Cellulose by Addition Polyvinyl Alcohol and Ability to Industrial Applications", M.Sc. thesis, Babylon University, College of Science, 2012 